\title{
Kaleidoskop der Geldwäschereivortaten: die strafgesetzliche Definition der Geldwäscherei und ihre Abwandlung durch die Rechtsprechung
}

\author{
Von Dr. Nadja Capus, Universität Basel
}

\section{Einführung}

Metaphorisch werden vielfältige, abwechslungsreiche Dinge als Kaleidoskop ${ }^{1}$ bezeichnet. Der Erfinder hat es profan „Schönbildschauer“2 genannt und als „natürliche Magie“ 3 bezeichnet.

Die Definition der Geldwäscherei und ihrer Vortaten hat selbstverständlich überhaupt nichts damit zu tun: weder mit Natur noch mit Magie. Vielfältigkeit und Abwechslungsreichtum sind zudem keine Attribute, die einen strafgesetzlichen Tatbestand auszeichnen sollten. Gleichwohl wird dieser Aufsatz in vier Schritten aufzeigen, was für ein erstaunlich vielfältiges und abwechslungsreiches Gebilde die schweizerische Geldwäschereikonzeption vor allem in Bezug auf ihre Vortaten ist. Dafür wird der Tatbestand der Geldwäscherei als ein vom Gesetzgeber gebautes Kaleidoskop betrachtet und aufgezeigt, aus welchen Elementen dieses gesetzgeberische Kaleidoskop besteht, welche anderen Akteure an der Konstruktion beteiligt sind, welch erstaunliche Bilder die Judikative beim Verwenden und Drehen offenbar gesehen hat und wie, weil diese nicht genügten, sogar noch neue Kaleidoskope erbaut worden sind. Von besonderer Bedeutung ist dabei der Einfluss, den richterliche Entscheide bezüglich rechtshilferechtlicher Gesuche auf die Auslegung des Tatbestands im Rahmen schweizerischer Strafverfahren haben.

1 Das Spielzeug wurde vom schottischen Physiker David Brewster 1816 erfunden - als Nebenprodukt seiner Forschung über die Brechung von Licht. Es besteht aus einem Rohr, das mit Spiegeln in der Innenseite und kleinen, farbigen Gegenständen zwischen zwei Glasplatten am einen Ende versehen ist. Am anderen Ende kann man hindurchsehen und beim Drehen des Rohres entstehen immer neue symmetrische, farbige Muster. Brewster, Letters on natural magic, addressed to Sir Walter Scott, Bart, London 1832.

2 Griechisch: Kaleidoskop, zusammengesetzt aus: kalós (schön), eĩdos (Bild), skopeĩn (betrachten, schauen).

3 Brewster (Anm. 1). 


\section{Geldwäschereidefinition des schweizerischen Strafgesetzgebers}

In der Schweiz sind Volk und Parlament die Gesetzgeber ${ }^{4}$. Da die Legislative deshalb von den drei Gewalten dem Volk und damit dem Souverän am nächsten steht, erscheint sie im Vergleich zur Judikative oder Exekutive als oberste Gewalt. Diese starke Position zeigt sich in der Ablehnung der Kontrolle der Legislative durch eine institutionelle Verfassungsgerichtsbarkeit ${ }^{5}$ oder auch in der Übertragung vieler Regierungs- und Verwaltungsfunktionen, aber auch richterlicher Aufgaben an die Legislative - die Schweiz ist beispielsweise im internationalen Vergleich ${ }^{6}$ eine Ausnahmeerscheinung darin, dass sie nicht nur das Amnestierecht ${ }^{7}$ dem Bundesparlament zugesteht, sondern auch das Begnadigungsrecht ${ }^{8}$ auf Bundes- wie auf Kantonsebene den Parlamenten gewährt⿳9.

Dieser starke Gesetzgeber hat 1990 den Tatbestand der Geldwäscherei ${ }^{10}$ eingeführt. Demnach droht der Gesetzgeber mit einer Freiheitsstrafe bis zu drei Jahren oder mit einer Geldstrafe, wenn jemand - und das kann eine natürliche oder seit Einführung der Unternehmensstrafbarkeit ${ }^{11}$ vor vier Jahren auch eine juristische Person ${ }^{12}$ sein - durch irgendeine Handlung einen der folgenden Strafverfolgungserfolge vereitelt:

- die Ermittlung der Herkunft von Vermögenswerten, die aus einem Verbrechen herrühren,

4 Bundeszuständigkeit zur Strafgesetzgebung: Art. 123 Abs. 1 BV; Zuständigkeit der Bundesversammlung: Art. 148 Abs. 1 und 163 Abs. 1 BV; Zuständigkeit des Volkes: Art. 138142 BV (Initiative und Referendum).

5 Letztmals im Rahmen der Totalrevision der Bundesverfassung (1995-2000) sowie im Rahmen der 1999 eingeleiteten Justizreform. Siehe zudem weiterführend: Müller, Die Verfassungsgerichtsbarkeit im Gefüge der Staatsfunktionen, VVDStrL 39 (1981), 55-95.

6 Lambert-Abdelgawad/Martin-Chenut, La prescription en droit international: vers une imprescriptibilité de certains crimes?, S. 101-161, in: Ruiz Fabri/della Morte/Lambert-Abdelgawad/Martin-Chenut (Hrsg.), La clémence saisie par le droit, amnistie, prescription et grâce en droit international et comparé, Paris 2007.

7 Art. 384 StGB.

8 Art. 381 StGB.

9 Capus, Ewig still steht die Vergangenheit? Der unvergängliche Strafverfolgungsanspruch nach schweizerischem Recht, Bern 2006, S. 24 f. Dazu das schweizerische Bundesgericht in BGE 84 IV 139, 141 f., bestätigt in BGE 118 Ia 104, 107. Außerdem: Lucco-Dénéréaz/Roth, La grâce, institution entre tradition et changements, Lausanne 1988; Real, Die Begnadigung im Kanton Aargau, Zürich 1981, S. 63; Schlatter, Die Begnadigung im Kanton Zürich, Zürich 1970, S. 31 ff.; Bruni, Die Begnadigung im Baselstädtischen Recht, Basel 1974, S. 37 ff. Die Meinung, dass der Gnadenakt materiell ein Judikativakt ist, wird vertreten von Dimoulis, Die Begnadigung in vergleichender Perspektive: rechtsphilosophische, Verfassungs- und strafrechtliche Probleme, 1996, S. 128 m.w.H. in Fn. 261.

10 Art. 305 bis StGB.

11 Art. 102 Abs. 1 und 2 StGB.

12 Selbst dann, wenn sie nicht dem verwaltungsrechtlichen Geldwäschereigesetz untersteht: Capus, Selbstregulierung: Ein taugliches Instrument zur Kontrolle der Geldwäscherei?, in: Capus/Cassani/Cimichella/Oberholzer (Hrsg.), Öffentlich - Privat. Neue Aufgabenteilung in der Kriminalitätskontrolle? Zürich 2006, S. 259-281. 
- die Auffindung von Vermögenswerten, die aus einem Verbrechen herrühren, - die Einziehung von Vermögenswerten, die aus einem Verbrechen herrühren.

„Verbrechen“ ist eine rechtliche Kategorie, für die eine Legaldefinition besteht: Gemäß Art. 10 Abs. 2 StGB sind Verbrechen diejenigen Taten, die der Gesetzgeber mit Freiheitsstrafen von mehr als drei Jahren bedroht hat. Die Verbindung „aus einem Verbrechen herrühren" muss zudem auch subjektiv gegeben sein: der Täter, die Täterin muss wissen oder zumindest annebmen, dass die Vermögenswerte aus einem Verbrechen stammen ${ }^{13}$.

Der Gegenstand des Geldwäschereitatbestands ist also so klar wie einfach: Sanktionierung der Tarnung von Vermögenswerten aus Verbrechen. Ansonsten ist der Gesetzestext aber in Vielem unklar, weil unbestimmt. Das liegt an der verwendeten Technik, denn der Gesetzgeber umschreibt nicht direkt die relevanten Tarnungshandlungen (wie beispielsweise die Unterbrechung der Papierspur, das Anlegen von Drogengeldern ${ }^{14}$ ), sondern bezieht die Inkriminierung nur auf die möglichen Konsequenzen allfälliger Tarnungshandlungen: die Vereitelung der Ermittlung und des Auffindens von Vermögenswerten aus Verbrechen. Dabei soll der tatsächliche Eintritt eines Vereitelungserfolgs bei diesem Delikt gegen die Rechtspflege ${ }^{15}$ unerheblich sein, die abstrakte Gefährdung des Strafverfolgungserfolgs bereits genügen ${ }^{16,17}$.

Gerechtfertigt wird diese Inkriminierung damit, dass ohne Kenntnis des Verbleibens der verbrecherischen Vermögenswerte einerseits die Einziehung durch die Strafverfolgungsbehörden und andererseits die Aufklärung der Vortat erschwert ist: „Hauptmotiv“ ist die Ermittlung des Täters der Vortat ${ }^{18}$. Mit den

13 Üblicherweise wird in solchen Fällen der Maßstab einer „Parallelwertung in der Laiensphäre“ (dazu Stratenwerth, Schweizerisches Strafrecht, Allgemeiner Teil I: Die Straftat, 3. Auflage Bern 2005, $\$ 9$ Rn. 70) angesetzt, wonach eine verbrecherische Vortat eine „schwerwiegende Vortat" sei, die „eine erhebliche Sanktion“ nach sich ziehe. Siehe BGE 119 IV 242 E. 2 a. Für einen Überblick der schweizerischen Rechtsprechung zur subjektiven Seite des Geldwäschereitatbestands siehe Pieth, StGB Art. 305bis StGB Rn. 46 ff., in: Niggli/Wiprächtiger (Hrsg.), Basler Kommentar, Strafgesetzbuch II, Basel 2007.

14 Vgl. dazu etwa BGE 119 IV 63 E. d oder 119 IV 243 f., 122 IV 213 E. 1, 2c (Umtausch von Geldscheinen in andere, größere Geldscheine), 122 IV 215 E. 2b (Verstecken fremden Drogenerlöses in der Wohnung von Drittpersonen), 126 IV 258 E. 2 (Entgegennahme von Drogenerlös zur Vermögensverwaltung).

15 BGE 129 IV 322 E. 2.2.4.

16 BGE 124 IV 276 E. 2, 128 IV 131 E. 7a.

17 Vgl. Vest, Probleme des Herkunftsprinzips bei der Geldwäscherei, in: Ackermann et al. (Hrsg.), Wirtschaft und Strafrecht, Festschrift für Niklaus Schmid, Zürich 2001, S. 417-437, 434: Eine bloße Behinderung der Einziehung soll ihm zufolge hingegen nicht genügen.

18 Botschaft über die Änderung des Schweizerischen Strafgesetzbuchs: Gesetzgebung über die Geldwäscherei und mangelnde Sorgfalt bei Geldgeschäften vom 12. Juni 1989, BBl 1989 II 1081; Egger Tanner, Die strafrechtliche Erfassung der Geldwäscherei, Zürich 1999, S. 13. An dieser Dualität des Interessenschutzes haben die Vereinten Nationen im Übereinkommen vom 15. November 2000 gegen die grenzüberschreitende organisierte Kriminalität (Palermo I) in Art. 6 Ziff. 1 (a) (i) und die Europäische Union in Art. 1 (2)(a) der Richtlinie 2005/60/EG des Europäischen Parlaments und des Rates vom 26. Oktober 2005 zur Verhinderung der 
Worten des Bundesgerichts: „Dass Schutzgut des Geldwäschereitatbestands in erster Linie das öffentliche Interesse an einem reibungslosen Funktionieren der Strafrechtspflege bildet, liegt außer Streit" ${ }^{19}$. Im Vordergrund hat dabei das spezielle Interesse der Strafrechtspflege an der Durchsetzung des staatlichen Einziehungsanspruchs zu stehen ${ }^{20}$. Der Lehrstreit, ob neben dem öffentlichen Interesse an der Vermögenseinziehung auch das private Interesse des durch die Vortat Geschädigten an einer Durchsetzung seines Rückerstattungsanspruchs geschützt sei, wurde vom Bundesgericht im Jahr 2003 zugunsten der Individualinteressen entschieden $^{21}$.

Die Entstehungsgeschichte des Delikts bestätigt die enge Verbindung zum Einziehungsrecht: eigentlich hätte gleichzeitig mit der Einführung des Geldwäscheartikels auch die strafrechtliche Einziehung revidiert werden sollen; dieses Revisionsprojekt ist dann aber abgetrennt und zeitversetzt als eigenes und als „Zweites Maßnahmepaket zur Bekämpfung des organisierten Verbrechens“ bezeichnetes Gesetzgebungsprojekt behandelt worden ${ }^{22}$.

Allerdings ist zu der von der Lehre vertretenen Sichtweise, dass der Geldwäschereitatbestand als Einziehungstatbestand konzipiert sei, zu präzisieren, dass der Geldwäschereitatbestand keinen eigenen Einziehungsanspruch kreiert. Sämtliche Ansprüche des Staates auf Einziehung von Vermögenswerten entstehen aufgrund von Art. 70 StGB lit. b und Art. 72 StGB, wobei der in Art. 70 verwendete Begriff der Straftat weiter geht als der Begriff „Verbrechen“ und auch Vergehen und Übertretungen sowie Zuwiderhandlungen gegen amtliche Verfügungen (nach Art. 292 StGB) umfasst ${ }^{23}$. Daraus folgt, dass mit dem Tatbestand der Geldwäscherei ein ganz bestimmter Einziehungsanspruch, nämlich derjenige auf Vermögenswerte, die aus einem Verbrechen herrühren, zusätzlich geschützt sein soll, indem er eben speziell dagegen gerichtete Handlungen - und nur diese - inkriminiert ${ }^{24}$.

Nutzung des Finanzsystems zum Zwecke der Geldwäsche und der Terrorismusfinanzierung, ABl. L 309/15, festgehalten.

BGE 129 IV 322, 326 E. 2.2.4

BGE 127 IV 79 E. 2e.

21 Vgl. BGE 120 IV 323, 327 E. 3c, in welchem das Bundesgericht noch keine eigene Stellung bezieht, und BGE 129 IV 322, 326 E. 2.2.4. (in welchem die adhäsionsweise von der Banca Popolare di Milano geltend gemachte Schadenersatzklage gemäß Art. 41 OR als zulässig erachtet). Zum Lehrstreit Cassani, Crimes ou délits contre l'administration de la justice: Art. 303-311 CP, in: Schubarth (Hrsg.), Kommentar zum Schweizerischen Strafrecht: Schweizerisches Strafgesetzbuch, Besonderer Teil, Bern 1996, Rn. 5; dies., Le blanchiment d'argent, un crime sans victime?, in: Ackermann et al. (Hrsg.) (Anm. 17), S. 393-415, 401, für den Schutz von Individualinteressen und m.w.H.

22 Ausführlich zur Entstehung - die zurück in die 1980er Jahre reicht - und Entwicklung der Geldwäschereibekämpfung in der Schweiz: Capus, Country Report: Combating Money Laundering in Switzerland, in: Pieth/Aiolfi (Hrsg.), A Comparative Guide to Anti-Money Laundering. A Critical Analysis of the Systems in Singapore, Switzerland, the UK and the USA, Cheltenham 2004, S. 114-226, 128 ff.

23 Schmid, StGB Art. 69 Rn. 27, Fn. 93, in: Schmid/Arzt (Hrsg.), Kommentar Einziehung, organisiertes Verbrechen, Geldwäscherei, 2. Auflage Band I, Zürich 2007.

24 Ackermann, StGB Art. 305 bis Rn. 54, in: Schmid/Arzt (Hrsg.) (Anm. 23), 1. Auflage Band II, Zürich 1998. 
Entsprechend wird der Tatbestand in der Literatur auch als Sekundärtatbestand bezeichnet, denn Geldwäscherei ist nach dieser Konzeption eine verselbstständigte Form der Beihilfe nach dem Verbrechen ${ }^{25}$. Diese Konzeption als Anknüpfungstatbestand verdeutlicht noch der Wortlaut der Ziffer 3 des Art. 305bis StGB, wonach der Täter ausdrücklich auch bestraft werden soll, wenn die „Haupttat" im Ausland begangen wurde und am Begehungsort strafbar ist. Folglich handelt es sich bei der Geldwäscherei um eine Nach- oder Nebentat.

Um dies an einem Beispiel zu illustrieren, das weiter unten wieder aufgenommen wird: Der Betrug nach Art. 146 StGB ist in Verbindung mit Art. 10 Abs. 2 StGB ein Verbrechen, da es möglich ist, Betrügern eine Gefängnisstrafe von bis zu fünf Jahren aufzuerlegen. Vermögenswerte, die aus einem Betrug herrühren, sind also geldwäschetangliche Tatobjekte.

Betrügereien gegenüber dem Staat im Bereich des Steuerrechts sind dagegen in der Schweiz traditionellerweise privilegierte Delikte. Diese Haltung hat der Gesetzgeber 1974 bei der Einführung des Verwaltungsstrafrechts (VStrR) gesetzlich verankert ${ }^{26}$. Entsprechend ist die herrschende Meinung der Lehre, dass

1. Art. 14 VStrR als Spezialgesetz dem Art. 146 StGB vorgeht, wenn jemand das Gemeinwesen um Abgaben betrügt, und dass es sich

2. selbst bei einer Urkundenfälschung (eigentlich ein Verbrechen ${ }^{27}$ ) zwecks Steuerbetrug nur um ein Vergehen handelt ${ }^{28}$.

Damit sind Fiskaldelikte keine geldwäschetauglichen Vortaten und kann der Tatbestand der Geldwäscherei nicht erfüllt sein, wenn das Geld einem Steuerbetrug entspringt: denn Vermögenswerte, die nicht aus einem Verbrechen stammen, können gar nicht erst Gegenstand einer Geldwäschehandlung sein ${ }^{29}$.

Fazit dieses ersten Teils ist also, dass nach der strafgesetzlichen Konzeption Geldwäscherei nur strafbar ist, wenn ein Verbrechen vorliegt und es zwischen den getarnten Vermögenswerten und diesem Verbrechen eine Verbindung gibt. Der Gesetzgeber hielt offensichtlich nicht jede Straftat für derart schwerwiegend, dass er das Verschleiern der daraus resultierenden Vermögenswerte hat extra sanktionieren wollen ${ }^{30}$ - selbst dann nicht, wenn die Geldwäscherei im Zusammenhang mit kriminellen Organisationen steht, welche ihr Geld ja nicht ausschließlich aus Verbrechen nach schweizerischem Strafrecht generieren, sondern auch aus Ver-

\section{Pieth bereits erzielt worden sein (Anm. 13, StGB Art. 305bis StGB Rn. 17).}

26 Botschaft des Bundesrates an die Bundesversammlung zum Entwurf eines Bundesgesetzes über das Verwaltungsstrafrecht vom 21. April 1971, Band 1, Heft 23, 993-1103, 1000.

27 Art. 251 StGB.

28 Pieth (Anm. 13), Rn. 13 f.; Arzt, StGB Art. 146 Rn. 139, in: Niggli/Wiprächtiger (Hrsg.) (Anm. 13); Boog, StGB Art. 251 Rn. 106 f., in: Niggli/Wiprächtiger (Hrsg.) (Anm. 13).

29 Deutlich in diesem Sinne das Bundesgericht im Urteil vom 24. Oktober 2005, 1 A.188/2005, E. 2.5 .

30 Botschaft 1989 (Anm. 18), 1082. 
gehen wie beispielsweise dem Zigaretten- oder Menschenschmuggel oder Warenfälschungen ${ }^{31}$.

\section{Die Geldwäschereikonzeptionen internationaler Organisationen}

Einzelstaatliche Gesetzgebungstätigkeit ist eingebettet in die internationale normative Rechtslage. Die Geldwäschereibekämpfung ist vor allem seit den 1990er Jahren internationalisiert. Auf dieser Ebene lassen sich zwei Phasen unterscheiden: In einer ersten Phase fand zwar eine Internationalisierung statt, gleichzeitig aber auch eine „Renationalisierung“, indem die erste Generation internationaler Übereinkommen gegen die Geldwäscherei es den Vertragsstaaten überließen, die Vortaten zur Geldwäscherei eigenständig zu bestimmen. Zu Beginn wurde der Geldwäschereibegriff von den Staaten - mit Ausnahme der Schweiz - mehrheitlich nur auf das Waschen von Erlösen aus Drogenstraftaten begrenzt ${ }^{32}$. Seit einigen Jahren aber wird der Begriff viel weiter gefasst, mit der Folge, dass nicht mehr nur Drogenhandel, sondern ein möglichst breites Spektrum von Straftaten Vortaten zu Geldwäschereihandlungen werden sollten. Mit diesem zweiten Trend hat der Spielraum für einzelstaatliche Vortatenkonzeptionen abgenommen: die meisten Übereinkommen listen heute selbst Dutzende von Delikten und Deliktskategorien auf, die als Vortaten zu gelten haben ${ }^{33}$.

31 Von Seiten des Autors des Vernehmlassungsentwurfs (1986), Bernasconi, bestand der Vorschlag, neben dem Herrühren der Vermögenswerte aus einem Verbrechen die Zurechenbarkeit zu einer kriminellen Organisation genügen zu lassen. Diese Variante wurde aber vom Parlament nicht angenommen, mit der wichtigen Folge, dass erstens im Gesetzestextes jegliche Verbindung zum ursprünglichen kriminalpolitischen Ziel des Gesetzgebers, mit der Geldwäschereikriminalisierung kriminelle Organisationen zu bekämpfen, fehlt und zweitens die - strafprozessual wichtige - Möglichkeit wegfällt, bei einem Geldwäschereivorwurf auf den Nachweis der Herkunft der getarnten Vermögenswerte aus einem Verbrechen zu verzichten und alternativ die Zugehörigkeit der Vermögenswerte zur Verfügungsmacht einer kriminellen Organisation nachzuweisen. Capus, (Anm. 22), S. 131; Tschigg, Die Einziehung von Vermögenswerten krimineller Organisationen, Bern 2003, S. 7 Fn. 12; Cassani, L'argent des organisations criminelles. A propos du deuxième train de mesures contre le crime organisé, in: Centre de droit bancaire et financier, Journée 1994 de droit bancaire et financier, Zürich 1994, S. 58

32 Botschaft 1989 (Anm. 18), 1073 f. und 1080; Capus (Anm. 22), S. 130 ff.

33 Vgl. Art. 3 Ziff. 4 und 5 lit. a-f der Richtlinie 2005/60/EG des Europäischen Parlaments und des Rates vom 26. Oktober 2005 zur Verhinderung der Nutzung des Finanzsystems zum Zwecke der Geldwäsche und der Terrorismusfinanzierung, ABl. L 309/15, sowie Art. 6 Ziff. 2 des Übereinkommens der Vereinten Nationen vom 15. November 2000 gegen die grenzüberschreitende organisierte Kriminalität (Palermo I).

Von Bedeutung sind insbesondere die Empfehlungen der FATF (Financial Action Task Force), einem zwischenstaatlichen Gremium mit 35 Mitgliedern, siehe http://www.fatf-gafi. org/document/52/0,3343,en_32250379_32237295_34027188_1_1_1_1,00.html, besucht am 1.12.2009. Die FATF-Empfehlungen sind vom Internationalen Währungsfonds und der Weltbank zum internationalen Standard zur Bekämpfung der Geldwäsche und der Finanzierung von Terrorismus postuliert worden. Siehe kritisch zur Rolle der FATF: Capus, Le droit pénal et la souveraineté partagée, Revue de science criminelle et de droit pénal comparé 2005, Nr. 2, 251-263. 
Die Rechtssetzungstätigkeit der EU, bzw. EG in Bezug auf die Geldwäscherei führte bisher zu drei Richtlinien und Rahmenbeschlüssen (1991, 2001, 2005). Sie verpflichten zwar nur die Mitgliedstaaten, sind aber dennoch für die Schweiz relevant, weil diese bilateral ein sogenanntes Betrugsbekämpfungsabkommen ${ }^{34}$ ausgehandelt hat. Dieses sieht eben nicht nur amts- und rechtshilferechtliche Zusammenarbeit bei Betrugsdelikten vor, sondern auch im Bereich der Geldwäsche und diese Zusammenarbeit spielt sich im Rahmen des materiellen Anwendungsbereichs der Richtlinien und der damit in Zusammenhang stehenden künftigen Rechtsprechung des Gerichtshofs der Europäischen Gemeinschaften ab ${ }^{35}$.

\section{Die vierte Gewalt}

Es liegt in der Regelungskompetenz von Regierung und Parlament, internationalrechtliche Übereinkommen sowie bi- und multilaterale Staatsverträge abzuschließen. Der Basler Völkerrechtler Luzius Wildhaber hat sie deshalb „treaty making power “36 genannt, denn längst lässt sich die Funktionsweise einer staatlichen Gemeinschaft nicht mehr wie zur Blütezeit des sogenannten Gesetzgebungsstaates im 19. Jahrhundert in das dreigliedrige Gewaltendreiteilungsdogma pressen ${ }^{37}$. Folg-

Ihre erste Empfehlung (1990), dass die Drogendelikte, wie sie im Übereinkommen der Vereinten Nationen gegen den unerlaubten Verkehr mit Suchtstoffen und psychotropen Stoffen vom 20. Dezember 1988 festgehalten sind, als Geldwäschevortaten zu gelten haben, wandelte sich in der 2003 revidierten Fassung zur Empfehlung, dass die Staaten das Verbrechen der Geldwäsche auf alle schweren Verbrechen unter Berücksichtigung einer weitgefassten Reihe von Vortaten anwenden sollten. Jedes Land sollte zumindest über eine größere Zahl von Verbrechen innerhalb der folgenden vorgegebenen Verbrechenskategorien verfügen: Teilnahme an einer organisierten kriminellen Gruppe sowie kriminellen Geschäften, Terrorismus einschließlich dessen Finanzierung, Menschenhandel und Einwandererschmuggel, Sexuelle Ausbeutung einschließlich sexueller Ausbeutung von Kindern, Unerlaubter Handel mit Betäubungsmittel und psychotropen Substanzen, Unerlaubter Waffenhandel, Hehlerei, Korruption und Bestechung, Betrug, Geldfälschung, Produktfälschung und -piraterie, Umweltverbrechen, Mord, schwere Körperverletzung, Entführung, unerlaubte Freiheitsberaubung und Geiselnahme, Raub oder Diebstahl, Schmuggel, Erpressung, Urkundenfälschung, Piraterie, Insider-Handel und Marktmanipulation.

34 Abkommen über die Zusammenarbeit zwischen der Schweizerischen Eidgenossenschaft einerseits und der Europäischen Gemeinschaft und ihren Mitgliedstaaten andererseits zur Bekämpfung von Betrug und sonstigen rechtswidrigen Handlungen, die ihre finanziellen Interessen beeinträchtigen (mit Schlussakte) (Abk.: BBA), SR 0.351.926.81.

35 Der Gerichtshof der Europäischen Gemeinschaft hat mit seiner Rechtsprechung den Zugriff der Gemeinschaftsorgane auf das Strafrecht erheblich beeinflusst. Vgl. beispielsweise das Urteil vom 23.10.2007 in der Rs. C-440/05, Klage betreffend Bekämpfung der Verschmutzung durch Schiffe mit Strafrecht und das Urteil vom 13.9.2005, Rs. C-176/03, Klage betreffend Umweltstrafrecht.

36 Wildhaber, Legalitätsprinzip und Aussenpolitik - eine Problemskizze, in: ders., Wechselspiel zwischen Innen und Aussen. Schweizer Landesrecht, Rechtsvergleichung, Völkerrecht, Basel 1996, S. 165-177.

37 Eichenberger, Zur Einleitung: Von der Rechtssetzungsfunktion im heutigen Staat, ZSR, NF Band 93, 1974 II. Halbband, 7-27 (7, 10, 16). 
lich hat der Zürcher Staatsrechtler Werner Kägi internationale Abkommen sogar als „vierte Gewalt“ neben Legislative, Judikative und Exekutive positioniert ${ }^{38}$.

Diese vierte Gewalt hat das zuvor erwähnte Betrugsbekämpfungsabkommen zwischen der Schweiz und den Mitgliedstaaten der Europäischen Union abgeschlossen, das sowohl zoll-, agrar- und steuerrechtliche Verstöße als auch Verstöße gegen Regelungen im öffentlichen Beschaffungswesen sowie solche im Bereich der Subventionen umfasst. Für eine Minderheit der Mitgliedstaaten der Europäischen Union ist es bereits in Kraft getreten ${ }^{39}$.

In Bezug auf die hier interessierende Geldwäschereithematik ist festzustellen, dass das Abkommen die gegenseitigen Rechtshilfe- und Auslieferungspflichten bezüglich ausländischer Geldwäschereiverfolgungen von Fiskaldelikten mit einschließt und damit aus schweizerischer Sicht folgendermaßen erweitert ${ }^{40}$ :

In Art. 2 Abs. 3 wird eine Art von Geldwäscherei rechtshilfefähig gemacht, die es in dieser Weise im Schweizer Strafrecht gar nicht gibt, denn danach soll das „Waschen der Erträge“ aus sämtlichen unter dieses Abkommen fallenden Handlungen zum Ergreifen strafprozessualer Zwangsmaßnahmen durch Schweizer Behörden für fremde Strafverfahren ermächtigen. Einzige Bedingung ist nur noch, dass die zugrunde liegenden Taten (also die Vortaten) nach dem Recht beider Vertragsparteien mit einer Freiheitsstrafe oder freiheitsbeschränkenden Maßregel der Besserung und Sicherung im Höchstmaß von mehr als sechs Monaten bedroht sind. Die Diskrepanz zum zuvor erwähnten, nach Schweizer Strafrecht verlangten Mindestmaß von drei Jahren ist offensichtlich. Um also die volle Wirksamkeit der EU-Rechtsnormen zu gewährleisten, werden mit dem Betrugsbekämpfungsabkommen für die Schweiz auch Handlungen zur rechtshilfefähigen Geldwäscherei, die aus nationaler strafrechtlicher Sicht aufgrund fehlenden Herrührens aus einer verbrecherischen Vortat keine Geldwäscherei sind. Das ist nur möglich, wenn die Rechtsnorm der beidseitigen Strafbarkeit aufgehoben wird, weshalb Art. 2 Abs. 2 des Abkommens statuiert, dass zumindest für den Bereich des Abkommens im Allgemeinen der Grundsatz der beidseitigen Strafbarkeit aufgehoben sei ${ }^{41}$.

38 Kägi, Das Problem der Gewaltenteilung im allgemeinen, in: Nawiasky (Hrsg.), Die Durchführung der Gewaltenteilung in der Schweiz, Einsiedeln, Köln 1949, S. 10-19; ders., Zur Entwicklung des Schweizerischen Rechtsstaates seit 1848, ZSR, NF Band 71 „Hundert Jahre schweizerisches Recht“, 1952.

39 Laut Bundesamt für Justiz seit 8. April 2009 für Bulgarien, Frankreich, Polen, Rumänien, Schweden; seit 9. April 2009 für Deutschland; seit 15. April 2009 für Finnland; seit 20. April 2009 für Großbritannien. Siehe http://www.rhf.admin.ch/rhf/de/home/straf/recht/multila teral/sr0-351-926-81.html, besucht am 1.12.2009.

40 Wobei vorweg anzumerken ist, dass die schweizerische Rechtsprechung dies ohnehin bereits eingeführt hatte, siehe dazu unten Abschnitt IV.

41 Umfassend zum rechtshilferechtlichen Erfordernis der beidseitigen Strafbarkeit: Capus, Strafrecht und Souveränität: Das Erfordernis der beidseitigen Strafbarkeit in der internationalen Rechtshilfe in Strafsachen, Habilitationsschrift, Basel 2010 (im Erscheinen). 


\section{Die Geldwäschereikonzeption in der Auslegung der Judikative}

Die Rechtssetzung hat kein Monopol auf rechtsschöpferisches Handeln ${ }^{42}$ : Die Gerichte sind in der Anwendung der Gesetze frei und nur dem Recht verpflichtet. Als maßgebendes Recht bezeichnet die Bundesverfassung sowohl die Bundesgesetze als auch das Völkerrecht ${ }^{43}$. Die Bindung an das Gesetz nimmt in der kontinentaleuropäischen Strafrechtslehre eine bedeutende Rolle ein und es wurde der Grundsatz entwickelt, dass die rechtsanwendenden Behörden weder in der Inkriminierung noch in der Bestrafung über den gesetzlich vorgegebenen Raum hinausgehen dürfen. Sie sind nicht befugt, neue Straftatbestände aufzustellen oder sonstige Eingriffe in die Rechte des Bürgers zu ermöglichen, denn nur der Gesetzgeber besitzt die dafür notwendige, unmittelbare demokratische Legitimation ${ }^{44}$.

\section{Im Rahmen schweizerischer Strafverfahren}

Die Erkenntnis, dass der Nachweis in Bezug auf die verbrecherische Herkunft der Vermögenswerte für die Strafverfolgungsbehörde außerordentlich mühsam sein kann, folgte rasch nach Inkrafttreten des Gesetzes. Es ist deshalb nicht verwunderlich, dass das Bundesgericht bereits 1994 das Kaleidoskop ein erstes Mal gedreht hat, um die Vollzugstauglichkeit des Straftatbestands zu erhöhen. Es hat kurzerhand beschlossen, dass der mühselige Nachweis einer Verbindung zwischen Herkunft und Verwertung nicht im Sinne des Gesetzgebers habe sein können ${ }^{45}$. In Korrektur eines Genfer Gerichtsentscheids entschied es deshalb, dass die Verbindung zwischen Vortat und Geldwäscherei bewusst schwach zu halten sei ${ }^{46}$. In der Lehre wurde daraufhin festgestellt, dass das Gericht damit dem Geldwäschereitatbestand das einzige Profilelement genommen hat ${ }^{47}$.

Festzuhalten ist allerdings, dass im konkreten Fall die Vortat dem Gericht durchaus bekannt war und es sich tatsächlich um ein Verbrechen gehandelt hat. Eigentliches Ziel des Bundesgerichts war es allein gewesen, sicherzustellen, dass Beschuldigte auch in Fällen wegen Geldwäscherei verurteilt werden sollen können, in denen es nicht gelingt, sie für die Vortat selbst schuldig zu sprechen. Es genügt

44 Noll, Zusammenhänge zwischen Rechtssetzung und Rechtsanwendung in allgemeiner Sicht, ZSR, NF Band 93, 1974 II. Halbband, 249-278, 276.

45 Vgl. in diesem Zusammenhang die von Noll festgestellte Tendenz der schweizerischen Lehre (im Gegensatz zur deutschen), bei Diskrepanzen meist den Vorrang des Zwecks zu betonen, wobei allerdings die Rechtsprechung, besonders die strafrechtliche, diesem postulierten "Methodenmonismus" nicht ausnahmslos gefolgt ist, Noll (Anm. 44), 261, $270 \mathrm{f}$.

46

47
BGE 120 IV 323, 328 E. 3d.

Cassani (Anm. 21), S. 64 f. 
also der Nachweis, dass die inkriminierten Vermögenswerte aus einer als Verbrechen qualifizierten kriminellen Handlung stammen ${ }^{48}$.

Im Jahr 2000 bestätigte das Bundesgericht denn auch explizit den akzessorischen Charakter des Geldwäschereitatbestands und hielt fest, dass neben dem Nachweis der Geldwäschereihandlung auch die Vortat selbst, wie das Herrühren der Vermögenswerte aus eben dieser Vortat, nachzuweisen seien ${ }^{49}$.

Anspruchsvoll ist in der Praxis natürlich diese strafgesetzliche Konstruktion nicht nur in Bezug auf den Nachweis der objektiven Seite, sondern auch in Bezug auf die subjektive Erfassung der Verbindung zwischen Vortat und Vermögenswerten beim Geldwäschetäter. Ihm muss nachgewiesen werden, dass er wusste oder hat annehmen müssen, dass die inkriminierten Vermögenswerte aus einem Verbrechen herrühren. Wer also beispielsweise glaubt, die Einziehung illegaler Vermögenswerte aus einem Insiderdelikt ${ }^{50} \mathrm{zu}$ vereiteln, handelt nicht tatbestandsmäßig, da Insiderdelikte keine Verbrechen sind. Kurios ist die Rechtsprechung zur versuchten Geldwäscherei in Bezug auf die Annahme untauglicher Versuche der Geldwäscherei: Wird nämlich beispielsweise ein Finanzintermediär der eventualvorsätzlichen Geldwäscherei beschuldigt, weil er aufgrund der Umstände von einer verbrecherischen Vortat hätte ausgehen sollen, wird er selbst dann bestraft, wenn sich im Laufe der Strafuntersuchung einwandfrei feststellen lässt, dass das Geld tatsächlich aus hinterzogenen Steuern stammt und nicht verbrecherisch erlangtes Geld ist. Dann liegt nämlich ein in der Schweiz grundsätzlich strafbarer untauglicher Versuch ${ }^{51}$ der Geldwäscherei vor ${ }^{52}$.

Die Problematik liegt hier insbesondere in der Auswahl der Indizien und ihrer Wertung, wenn das Bundesgericht als Indiz für die verbrecherische Herkunft die beruflichen und finanzielle Situation des Vermögensinhabers oder auch nur die Höhe des Vermögenswertes genügen lässt ${ }^{53}$.

Wenn nun aber wirklich die Höhe des Betrags ein zulässiges Kriterium dafür sein soll, dass das Geld aus einem Verbrechen stammt und damit geldwäschetaugliches Tatobjekt ist, geht dies über eine einfache Drehung des bestehenden Kaleidoskops hinaus; der Gesetzgeber sollte den Geldwäschereitatbestand revidieren

Das Gericht hält im gleichen Entscheid (BGE 120 IV 328 E. 4) fest, dass bei Vorliegen von Vorbereitungshandlungen mit Blick auf die Tarnung der aus dem Verbrechen erwarteten Vermögenswerte eine versuchte Geldwäscherei angenommen werden kann, auch wenn die Vortat selbst noch nicht begangen worden ist.

49 BGE 126 IV 255, 261, E. 3a.

50 Art. 161 StGB (Ausnützen der Kenntnis vertraulicher Tatsachen).

51 Art. 22 Abs. 1 StGB.

52 Vgl. das Bundesgerichtsurteil 6S.769/1999 vom 7. März 2000 E. 4: „Le Tribunal fédéral a ainsi récemment admis qu'il pouvait y avoir délit impossible de blanchissage d'argent (Art. 305bis $\mathrm{CP})$ si l'auteur, à tort, envisage la possibilité que l'argent provienne d'un crime et s'en accommode (arrêt non publié du 24 février 1999, 6S.66/1999)“. Siehe Kritik und Hinweis auf ebensolche kantonalen Urteile bei Pieth (Anm. 13), Rn. 46c. 
und statt des qualitativen Kriteriums (Verbrechen) ein quantitatives (Betragshöhe) einfügen. Im selben Urteil (s. Anm. 53) hat das Bundesgericht diesen Ansatz noch relativiert und zugegeben, „...dass aus der Höhe des Deliktsbetrags allein nicht auf ein Verbrechen geschlossen werden kann. Beispielsweise stellt die einfache Veruntreuung auch dann ein Vergehen dar, wenn Hunderttausende von Franken veruntreut wurden “54.

\section{Im Rahmen von Verfahren der internationalen Rechtshilfe in Strafsachen}

Wenn das Bundesgericht über rechtshilferechtliche Belange zu entscheiden hat und hier tritt ein wichtiger Aspekt der weiteren Wandlung des gesetzlichen Geldwäschereitatbestands hinzu -, hat es die Tendenz, Tatbestände des Strafgesetzbuchs deutlich weiter auszulegen als in schweizerischen Strafverfahren.

Dies liegt erstens daran, dass die Judikative allgemein bei der Auslegung von internationalen Verträgen dazu neigt, sich an der Meinung der Exekutive zu orientieren ${ }^{55}$. Zweitens liegt es an der Kombination der zentralen rechtshilferechtlichen Rechtsnorm der beidseitigen Strafbarkeit mit dem Günstigkeitsprinzips, welches im internationalen Rechtshilferecht allgemein gilt ${ }^{56}$. Mit der Rechtsnorm der beidseitigen Strafbarkeit nimmt die Schweiz für sich in Anspruch, Strafverfahren anderer Staaten nur dann mit ihren strafprozessualen Zwangsmaßnahmen zu unterstützen oder Personen auszuliefern, wenn der im Ausland erhobene Vorwurf ein Verhalten betrifft, das - hätte es sich in der Schweiz abgespielt - nach schweizerischem Strafrecht inkriminiert wäre ${ }^{57}$. Bis zum Inkrafttreten des Betrugsbekämpfungsabkommens forderten sämtliche von der Schweiz abgeschlossenen Auslieferungsverträge die beidseitige Strafbarkeit. Im Bereich der akzessorischen oder kleinen Rechtshilfe konnte sich das Erfordernis der beidseitigen Strafbarkeit nicht in gleichem Maße durchsetzen. Dennoch sieht auch hier Art. 64 Abs. 1 IRSG vor, dass in Fällen, in denen rechtshilfeweise um die Vornahme strafprozessualer Zwangsmaßnahmen ersucht wird, die im Ausland verfolgte Handlung - hätte sie sich in der Schweiz ereignet - die objektiven Merkmale eines Straftatbestands erfüllen müsste. Seit einem bundesgerichtlichen Entscheid im Jahr 1986 ist zudem nach herrschender Lehre und Praxis auch der subjektive Tatbestand zu Bernasconi, La trasmissione di mezzi di prova dalla $\mathrm{S}$ Tendenze recenti, in: Bernasconi et al. (Hrsg.), Assistenza giudiziaria internazionale in materia civile, penale, amministrativa ed esecutiva, Lugano 1999, S. 59-115, 63: “[...] l'ideaguida dell'evoluzione giurisprudenziale relativa al principio di doppia punibilità [è] tesa risolutamente ad una interpretazione estensiva, allo scopo di evitare che l'applicazione del principio possa condurre ad una limitazione dell'assistenza."

57 
prüfen ${ }^{58}$. Die Gerichte müssen also auch in Rechtshilfefällen das Kaleidoskop des schweizerischen Gesetzgebers benutzen ${ }^{59}$.

Die Tendenz zur ausweitenden Auslegung manifestiert sich besonders deutlich in den bundesgerichtlichen Rechtshilfeentscheiden bezüglich ausländischer Geldwäschereivorwürfe. ${ }^{60}$ Unter Respektierung der strafgesetzlich vorgesehenen Bedingung, dass Geldwäsche eine verbrecherische Vortat voraussetzt, aber in $\mathrm{Ab}$ weichung zur materiellen Rechtslage in Bezug auf Fiskaldelikte ${ }^{61}$, haben das Bundesgericht und das Bundesstrafgericht (das seit der Revision des Rechtspflegegesetzes vor wenigen Jahren erstinstanzlich zuständig ist) das Feld möglicher Vortaten erweitert. Es ist eingangs dargelegt worden, dass aufgrund des schweizerischen Strafgesetzbuchs und des Verwaltungsstrafrechts Fiskaldelikte keine Geldwäschereivortaten sein können, da sie nur Vergehen sind. Nun hat aber das Bundesgericht bei Rechtshilfegesuchen, welche Geldwäschereivorwürfe enthalten, in Bezug auf

BGE 112 Ib 576 E. 4b; Zimmermann, La coopération judiciaire internationale en matière pénale, 3. Auflage Bern 2009, S. 537 Rn. 584.

59 Ausführlich dazu: Capus (Anm. 41), S. 225 ff. Die nachfolgenden Ausführungen sind teilweise dieser Arbeit entnommen.

60 Vgl. für weitere Beispiele: Bernasconi (Anm. 56), S. 63-69. Vor der Revision der Bundesrechtspflege konnte noch vermutet werden, dass diese ausweitende Auslegung dadurch begünstigt wurde, dass nicht der Kassationshof, sondern die verwaltungsrechtliche Abteilung des Bundesgerichts über Rechtshilfebelange entschieden und dass dies zu divergierenden Auslegungen strafrechtlicher Tatbestände geführt hat. Seither ist jedoch die Beschwerdekammer des Bundesstrafgerichts zuständig und die Tendenz ungebrochen.

61 Die schweizerische Amts- und Rechtshilfe in Fiskalsachen unterliegt aktuell einem tiefgreifenden Wandel, insbesondere gegenüber den USA und den Mitgliedstaaten der EU. Zwar sah bereits 1978 das Zusatzprotokoll zum Europäischen Übereinkommen über die Rechtshilfe in Strafsachen eine Ausweitung der Rechtshilfe auf Fiskaldelikte vor, dieses war aber von den damaligen Vertragsparteien (Frankreich, Deutschland, Belgien, Luxemburg und den Niederlanden) nur gerade von den Niederlanden ratifiziert worden. Nun verpflichtet das Schengener Durchführungsübereinkommen die Vertragsparteien in Artikel 50 zur Leistung von Rechtshilfe auch für Delikte im Bereich abschließend aufgezählter indirekter Steuern (Verbrauchssteuern, Mehrwertsteuern und Zollabgaben). Sollte die EU im Rahmen der Weiterentwicklung des Schengen-Acquis auch bei der internationalen Kooperation im Bereich der direkten Fiskalität das Erfordernis der beidseitigen Strafbarkeit abschaffen wollen, würde der Schweiz eine „unbefristete Ausnahme“ vom Grundsatz gewährt, dass die Ablehnung eines neuen Erlasses im äußersten Fall die Kündigung der Abkommen zur Folge hat. Konkret geht es um die Bekämpfung des (Zigaretten-)Schmuggels, der Geldwäscherei und des Subventionsbetrugs: in Bezug auf Abgabebetrug oder gewerbsmäßigen Schmuggel war die schweizerische Kooperation - auch die Amtshilfe im Zollbereich - ungenügend, da beide nach schweizerischem Strafrecht keine Vortaten der Geldwäscherei sind. Allerdings haben sich die Verhandlungen seit Beginn (2001) zwischen der Schweiz und der Europäischen Kommission nicht nur auf den Zigarettenschmuggel beschränkt, sondern bezogen sich auf „alle vorstellbaren Fälle von Verstößen gegen die finanziellen Interessen der Vertragsparteien“. Siehe Botschaft zur Genehmigung der bilateralen Abkommen zwischen der Schweiz und der Europäischen Union, einschließlich der Erlasse zur Umsetzung der Abkommen („Bilaterale II“) vom 1. Oktober 2004, BBl 2004 Band 1, 5965-6300, 5968, 6185; Koller, Schengen/Dublin und die Bilateralen II - eine neue Dimension in der Integrationspolitik. Vom einheitlichen Wirtschafts- zum europäischen Sicherheitsraum, Aktuelle juristische Praxis 14/2005, 909917, 916. 
- ungerechtfertigte Rückforderungen ausländischer Quellensteuern,

- ungerechtfertigte Rückerstattungen ausländischer Umsatzsteuern sowie

- ungerechtfertigte ausländische Rückerstattungsauszahlungen unter Anwendung von Mehrwertsteuerkarussellen

mehrmals entschieden ${ }^{62}$, dass ein solcher Betrug in Fiskalsachen nicht wie im innerstaatlichen Verhältnis unter das privilegierende Spezialgesetz falle, sondern unter den gemeinrechtlichen Tatbestand des Betrugs. Damit ist - im rechtshilferechtlichen Kontext - eine verbrecherische Vortat gegeben und es können zugunsten einer ausländischen Strafverfolgung der Geldwäscherei von Vermögenswerten aus Fiskaldelikten auf schweizerischer Seite sowohl Zwangsmaßnahmen ergriffen als auch Täter ausgeliefert werden.

Zudem brauchen nach geltender Rechtsprechung ausländische Rechtshilfeersuchen wegen des Verdachts der Geldwäscherei nicht notwendig zu erklären, worin die Vortat (Haupttat) besteht. Es genügt, wenn verdächtige Finanztransaktionen dargelegt werden: „Contrairement à ce que soutient la recourante, cette dernière notion se rapporte uniquement à l'infraction de blanchiment, telle qu'elle est définie à l'art. $6 \mathrm{CBl}$, et non aux agissements délictueux qui l'ont précédée: ceux-ci sont en effet définis à l'art. 1 let. e CBl, sous l'appellation spécifique d'infraction principale' (Haupttat). Ainsi, lorsqu'elle soupçonne une activité de blanchiment et sollicite l'entraide judiciaire à cet effet, l'autorité requérante n'a pas à indiquer en quoi consisterait l'infraction principale (arrêt du Tribunal fédéral 1 A.245/1996 du 6 décembre 1996, consid. 4b)“63. Dies hat das Bundesgericht in verschiedenen in der amtlichen Sammlung nicht veröffentlichten Entscheidungen bestätigt ${ }^{64}$.

Bundesstrafgericht, RR.2007.106, Entscheid vom 19. November 2007, II. Beschwerdekammer E. 3.3.; BGE 110 IV 24 E. 2e; Urteil des Bundesgerichts 1 A.194/2005 vom 18. August 2005, E. 3.1.4. Gemäß dieser Rechtsprechung sind die Spezialbestimmungen des Verwaltungsstrafrechts mit ihren milderen Strafandrohungen einschränkend auszulegen. Nach gemeinrechtlichem Betrug ist strafbar, wer sich aus eigener Initiative entschließt, sich oder Dritte durch Irreführung der Behörden unrechtmäßig zu bereichern, indem er auf raffinierte Weise fiktive fiskalische Rückerstattungsansprüche existierender oder erfundener Personen geltend macht und (mittels unechter oder unwahrer Urkunden) die Auszahlung der Rückerstattungsansprüche erwirkt. Es kann nach dieser Rechtsprechung nicht gerechtfertigt sein, einen solchen Fall von vorsätzlicher und arglistiger Vermögensschädigung des Staates unter deliktischer Ausnützung eines ausländischen fiskalischen Rückerstattungssystems zu privilegieren und von der Strafdrohung für gemeinrechtlichen Betrug auszunehmen (vgl. 1 A.189/ 2001 vom 22. Februar 2002, E. 5; 1 A.189/2002 vom 28. Oktober 2002, E. 2 und 3; 1 A.297/ 2005 vom 13. Januar 2006, E. 3).

63 BGE 129 II 97 E. 3.2, S. 99 (bestätigt in: 1 A.189/2006, Urteil vom 7. Februar 2007).

64 U.a. in den Entscheiden: 1 A.188/2005 vom 24. Oktober 2005 E. 2.2 mit Verweis auf: Urteile 1 A.295/2004 vom 27. Januar 2005 E. 3.2; 1 A.175/2004 vom 25. November 2004 E. 2.4 und 2.8; 1 A.141/2004 vom 1. Oktober 2004 E. 2.; 1 A.151/2004 vom 2. August 2004 E. 4.2; 1 A.231/2003 vom 6. Februar 2004 E. 5.3; 1 A.154/2003 vom 25. September 2003 E. 3.3 und 5. 
Diese Haltung des Bundesgerichts hat Kritik provoziert, denn tatsächlich stellt sich seither die Frage, ob die verbrecherische Vortat nun noch Definitionsmerkmal der Geldwäscherei ist oder nicht ${ }^{65}$. Interessanterweise scheint das Bundesgericht davon auszugehen, dass es bei Rechtshilfefällen ein neues Kaleidoskop in Händen hält - und begründet dies damit, dass sich gemäß Geldwäschereiabkommen des Europarats ${ }^{66}$ die Rechtshilfeleistung ausschließlich auf die geldwäschereiverdächtigen Transaktionen beziehe und keineswegs auf die Vortaten - weshalb die Vortaten bei einer Rechtshilfeleistung zum Geldwäschereivorwurf auch außer Acht zu lassen $\operatorname{seien}^{67}$.

Angesichts der Tatsache, dass der Europarat mit dem Geldwäschereiübereinkommen keineswegs das Ziel verfolgt hat, neue Normen und Konzepte zur Geldwäscherei festzuschreiben, ist diese Argumentation jedoch nicht schlüssig. Das Bundesgericht übernahm lediglich die zwei in den diversen staatlichen Gesetzen bereits gängigen Elemente zur Definition von Geldwäscherei: inkriminierte Vermögenswerte aus Vortaten einerseits und verschleiernde, verbergende Transaktionen und Handlungen andererseits. Der erwähnte Art. 1 lit. e definiert nicht etwa die Vor- oder Haupttaten der Geldwäscherei separat, wie das Bundesgericht anzunehmen scheint, sondern enthält schlicht die Begriffserklärung, dass der Ausdruck „Haupttat“ Straftaten meint, durch die Erträge erlangt wurden, die dann Gegenstand einer Geldwäschehandlung werden können ${ }^{68}$. Die Schweiz hat denn auch einen Vorbehalt zum Abkommen von 1990 angebracht und klargestellt, dass sie die Pflicht zur Kriminalisierung der Geldwäscherei nur in Bezug auf Straftaten anerkennen kann, die nach schweizerischem Recht ein Verbrechen darstellen ${ }^{69}$. Man mag umgangssprachlich alle möglichen Handlungen als Geldwäscherei bezeichnen; die Trennung aber von Vortat und Geldwäschereihandlung widerspricht der strafgesetzlichen Konzeption des Geldwäschereitatbestands. Darin zumindest war der Gesetzgeber klar: Geldwäscherei im Sinne des strafrechtlichen Tatbestands existiert überhaupt nur, wenn die Vermögenswerte aus einer verbrecherischen Vortat stammen.

Die Kritik hat das Bundesgericht dazu veranlasst, seine Position näher darzulegen. Es hat die eigenen Entscheide analysiert, interpretiert und verkündet, dass es

Harari, L'évolution récente en matière d'entraide pénale: des interrogations demeurent, in: Gani (Hrsg.), Récents développements en matière d'entraide civile, pénale et administrative, Lausanne 2004, S. 103-141, $123 \mathrm{f}$.

66 Übereinkommen über Geldwäsche sowie Ermittlung, Beschlagnahme und Einziehung von Erträgen aus Straftaten, Straßburg 1990, SEV 141/SR 0.311.53.

67 Forster, Internationale Rechtshilfe bei Geldwäschereiverdacht, Entwicklung und Typologie der bundesgerichtlichen Praxis zur Konkretisierung der verbrecherischen Vortat, ZStrR 124 (2006) 274 ff. m.w.H.

68 Siehe Art. 1 lit. e sowie den Vorbehalt der Schweiz zu Art. 6 und Art. 18 Ziff. 1 lit. f.

69 Vorbehalt der Schweiz zu Art. 6: „Artikel 6 Ziffer 1 des Übereinkommens findet ausschließlich Anwendung, wenn die Haupttat nach schweizerischem Recht ein Verbrechen darstellt (Art. 9 Abs. 1 StGB in Verbindung mit den Straftatbeständen des Strafgesetzbuchs und des Nebenstrafrechts).“ 
seine Urteile folgendermaßen differenzierend verstanden wissen will: Einerseits sei die „abstrakte Möglichkeit“, dass das Geld von einem Verbrechen herrühre, nicht genügend. Andererseits seien aber dann keine näheren Angaben zur Vortat erforderlich, wenn „erhebliche Indizien“ dafür bestünden, dass es sich dabei um ein Verbrechen handelt ${ }^{70}$.

Dafür wird u.a. wieder auf das quantitative Kriterium gegriffen, denn laut Bundesgericht liegt es nahe - und das selbst dann, wenn die ausländische Strafverfolgungsbehörde die Vortat nicht einmal ansatzweise zu schildern vermag ${ }^{71}$, , von einer außerordentlich schwerwiegenden Straftat und somit nach schweizerischem Recht von einem Verbrechen auszugehen, wenn es um Transaktionen von Millionen oder gar Milliarden von Franken geht und international zahlreiche Firmen oder Personen involviert sind. Außerdem werden Ehefrauen, Töchter und Söhne, die Geld erhalten oder Liegenschaften kaufen, zu erheblichen Indizien dafür, dass das Geld aus einem Verbrechen stammt. Selbst geographische Kriterien genügen als Indiz, dass ein Verbrechen vorliegt, denn nach Meinung des Bundesgerichts hat dies dann zu gelten, wenn das Geld auch nur teilweise aus Gegenden stamme, „die unter der organisierten Kriminalität bekanntermaßen leiden“. Auch die Benutzung von „Off-Shore-Gesellschaften“ bei den Transaktionen lassen nach der Rechtsprechung verbrecherische Vortaten vermuten ${ }^{72}$.

Diese Rechtsprechung zeigt in aller Deutlichkeit, wie prudentielle Kriterien also aufsichtsrechtliche Kriterien, die von den staatlichen Aufsichtsbehörden, den privaten Selbstregulierungsorganisationen, den Compliance-Abteilungen der Finanzinstitute in Reaktion auf den Kriminalstraftatbestand erlassen worden sind ${ }^{73}$,

Der Entschärfung der Spannung, welche zwischen dem. Derafischer Strafverfolgungsbehörden einerseits und der Konkretisierungsanforderung bei Rechtshilfeersuchen andererseits entsteht, dient zudem Art. 67a IRSG: die unaufgeforderte Rechtshilfe. Demnach können schweizerische Behörden einem potentiell interessierten Staat die Existenz von möglicherweise verbrecherisch erlangten Vermögenswerten in der Schweiz mitteilen und damit die ausländischen Strafverfolgungsbehörden über Details informieren, die in einem allfälligen Rechtshilfeersuchen an die Schweiz zu stehen hätten. Vgl. Bernasconi, Die Strafbehörden angesichts der Internationalisierung der Wirtschaftskriminalität, ZSR 104 (1985) II, 339 ff., 346. Dabei geht die schweizerische Regelung über die Übermittlung von Informationen hinaus und erlaubt sogar die spontane Übermittlung von Beweismitteln, wenn diese keinen Geheimbereich betreffen (wobei die Unterscheidung zwischen bloßer Information und Beweismittel unklar ist). Zimmermann, La coopération judiciaire internationale en matière pénale, 3. Auflage Bern 2009, S. 382 f.; Beglinger, Rechtshilfeverfahren: Anwesenheit, spontane Übermittlung und Zweites Zusatzprotokoll zum Europäischen Rechtshilfeübereinkommen, AJP 2007, $916 \mathrm{ff}$.

72 BGE 1 A.188/2005, Urteil vom 24. Oktober 2005, E. 2.4. und 2.5.

73 Vgl. beispielsweise die Verordnung der Eidgenössischen Bankenkommission vom 18. Dezember 2002 zur Verhinderung von Geldwäscherei, Art. 7 Geschäftsbeziehungen mit erhöhten Risiken:

1. Der Finanzintermediär entwickelt Kriterien, welche auf Geschäftsbeziehungen mit erhöhten Rechts- und Reputationsrisiken hinweisen.

2. Als Kriterien kommen je nach Geschäftsaktivitäten des Finanzintermediärs insbesondere in Frage: a. Sitz oder Wohnsitz der Vertragspartei und des wirtschaftlich Berechtigten oder 
um solche Straftaten zu verhindern - plötzlich zu Kriterien werden, um den strafrechtlichen Nachweis einer verbrecherischen Vortat zu erbringen. So ist ein neues Kaleidoskop entstanden, das verwendet wird, wenn es um Rechtshilfe zur Unterstützung ausländischer Strafverfahren geht.

3. Im Rahmen schweizerischer Strafverfahren unter Einfluss der Rechtsprechung in Rechtshilfeverfahren

Allerdings geschieht dies nicht nur im Rahmen von Rechtshilfeverfahren, denn rechtshilferechtlich neu entstandene Kaleidoskope werden auch in schweizerischen Strafverfahren verwendet. Dies illustriert im Zusammenhang mit der Geldwäsche ${ }^{74}$ ein Bundesgerichtsurteil vom Mai 2007, in dem das Gericht über die Verhältnismäßigkeit einer Kontosperre und Beschlagnahme des Geldes im Rahmen eines schweizerischen Strafverfahrens zu entscheiden hatte und dabei Bemerkenswertes festhielt: „Der Tatbestand der Geldwäscherei (Art. 305bis StGB) enthält zwei voneinander unabhängige Elemente: die Vereitelungshandlung einerseits und die Vortat anderseits. Jedenfalls in einer ersten Phase der Strafuntersuchung genügt es, dass ein hinreichender Tatverdacht bloß bezüglich eines der beiden Elemente besteht, es jedoch aufgrund der besonderen Umstände im Sinne eines Anfangsverdachts naheliegt, dass auch das andere Tatbestandsmerkmal erfüllt sein dürfte. In der Regel liegen den Untersuchungsbehörden zu Beginn eines Strafverfahrens bloß konkrete Anhaltspunkte zur Vereitelungshandlung vor. Als geldwäschereiverdächtig können namentlich Finanztransaktionen angesehen werden, bei denen hohe Beträge ohne erkennbaren wirtschaftlichen Grund und über Konten zahlreicher Gesellschaften in verschiedenen Staaten transferiert werden (vgl. BGE 129 II 97 E. 3.3 S. 100; unveröffentlichte Urteile 1 A.280/2005 vom 7. März 2006, E. 2.2.2 und 1 A.189/2006 vom 7. Februar 2007, E. 2.5)“75.

Die vom Bundesgericht zitierten Entscheide sind ausschließlich Rechtshilfeentscheide. Damit hat sich offensichtlich die neue, rechtshilferechtliche Kreation der Geldwäschereikonzeption auch für schweizerische Strafverfahren durchgesetzt.

deren Staatsangehörigkeit; b. Art und Ort der Geschäftstätigkeit der Vertragspartei und des wirtschaftlich Berechtigten; c. Fehlen eines persönlichen Kontakts zur Vertragspartei sowie zum wirtschaftlich Berechtigten; d. Art der verlangten Dienstleistungen oder Produkte; e. Höhe der eingebrachten Vermögenswerte; f. Höhe der $\mathrm{Zu}$ - und Abflüsse von Vermögenswerten; g. Herkunfts- oder Zielland häufiger Zahlungen.

3. Als Geschäftsbeziehungen mit erhöhten Risiken gelten in jedem Fall diejenigen mit politisch exponierten Personen.

74 Als weiteres Beispiel für den Einfluss rechtshilferechtlicher Rechtsprechung auf Strafrechtsentscheide ließe sich die Rechtsprechung zum Begriff „kriminelle Organisation“ anführen, welche maßgeblich auf die in Rechtshilfefällen entwickelte Auslegung des Begriffs abstellt (BGE 132 IV 134 f.).

75 BGE 1P.64/2007, Urteil vom 29. Mai 2007, Staatsrechtliche Beschwerde gegen Zwischenentscheid im Strafverfahren, E. 5 und 8. 


\section{Schlussfolgerung}

Anhand dieser Entwicklung lässt sich eine Verselbstständigung der Figur der Geldwäsche, eine Loslösung vom Unrecht der Vortat feststellen ${ }^{76}$ und es ließe sich folgern, dass eine Neudefinition des Geldwäschereitatbestands unumgänglich sei. Schließlich hat der Gesetzgeber zur Kenntnis zu nehmen, ob und wie sich das angestrebte Ziel tatsächlich mit der erlassenen Norm verwirklichen lässt ${ }^{77}$. Insofern würde die Erfahrung des richterlichen und verwaltenden Alltags zurückwirken auf die Rechtssetzung, um die erforderliche Vollzugstauglichkeit herzustel$1 \mathrm{en}{ }^{78}$

Ein solcher Eingriff steht aber, soweit absehbar, nicht auf der Handlungsliste des Gesetzgebers. Zur Zeit wird der Tatbestand nicht neu definiert, aber sein Geltungsbereich stetig ausgeweitet, indem der Gesetzgeber neue Verbrechen und damit neue Vortaten kreiert und die „vierte Gewalt“ neben der strafrechtlichen eine rechtshilferechtliche Geldwäscherei schafft. Die Rechtsprechung ihrerseits berücksichtigt vornehmlich polizeiliche und außenpolitische Interessen und setzt damit die Rechtssicherheit und die sie konkret bestimmenden Rechtsschutzinteressen wie das Prävisions- und das Legitimationsinteresse aufs Spiel ${ }^{79}$.

Auf diese Weise kommt die Schweiz auf pragmatische Weise ihren internationalen Verpflichtungen nach und schafft die Voraussetzungen für die Durchsetzung der Verträge und Abkommen im eigenen Land. Aber ein Rechtssystem, das langfristig kohärent sein will, wird um eine gesetzgeberische Neufassung der Definition nicht herumkommen, wenn die Rechtsordnung für ihre Straftaten ein fest definiertes Kaleidoskop haben soll. Einer vertieften Diskussion bedarf zudem die Frage, ob zur Kohärenz der Rechtsordnung nicht auch gehört, dass nur für diese Straftaten rechtshilfeweise strafprozessuale Zwangsmaßnahmen geleistet werden.

Pieth, „Korruptionsgeldwäsche“, in: Ackermann et al. (Hrsg.) (Anm. 17), S. 437-456, 438. Artikel 170 der neuen Bundesverfassung verlangt von der Bundesversammlung, dass sie dafür sorgt, dass die Maßnahmen (Gesetze, Verordnungen) des Bundes auf ihre Wirksamkeit überprüft werden. Dieser Auftrag betrifft unmittelbar das Parlament, mittelbar aber auch den Bundesrat und die Bundesverwaltung.

Eichenberger (Anm. 37), 19.

Siehe generell zur Folgenberücksichtigung im Rahmen teleologischer Interpretation Puppe, Kleine Schule des juristischen Denkens, 2008, S. 85 ff.; zu den Rechtsschutzinteressen, die den Inhalt der Rechtssicherheit bestimmen: Noll (Anm. 44), S. $273 \mathrm{ff}$. 